Rev Biomed 2005; 16:95-98.

\title{
Cirugía de doble switch, una alternativa segura y novedosa para la corrección de la levo- transposición de grandes arterias.
}

Caso clínico

Álvaro Durán, Víctor R. Castillo, Álvaro Montero, Cristina Villa-Roel.

Departamento de Cardiocirugía Pediátrica, Fundación Cardiovascular de Colombia, Floridablanca, Santander, Colombia.

\section{RESUMEN.}

Introducción. La levo-transposición de grandes arterias (L-TGA) es una cardiopatía congénita con pocas probabilidades de opción quirúrgica en nuestro medio. Dentro de las alternativas terapéuticas para su corrección, la cirugía de doble switch (CDS) ofrece una corrección fisiológica y segura para el infante en el corto y mediano plazo.

Casos clínicos. Se presentan dos casos de L-TGA con insuficiencia tricuspídea severa: uno de ellos asociado a anomalía de Ebstein. En ambos pacientes se realiza cerclaje de la arteria pulmonar como preparación a la CDS.

Comentario. La CDS se considera una alternativa segura y novedosa, ya que además de ofrecer una corrección anatómica y funcional de la L-TGA, evita los problemas asociados a la corrección clásica. Los dos casos presentados son los primeros realizados exitosamente en la Fundación Cardiovascular de Colombia (Bucaramanga-Colombia). Sin embargo, requieren de seguimiento en el largo plazo con especial énfasis en el comportamiento de los gradientes obstructivos de la aurícula derecha y de la insuficiencia tricuspídea que pueden llevar a signos de congestión venosa. (Rev Biomed 2005; 16:9598)

Palabras clave: Levo-transposición de grandes arterias, cirugía de doble switch.

\section{SUMMARY.}

Double switch surgery a safe and new alternative for the correction of levotrasposition of great arteries.

Introduction. The levo-transposition of the great arteries (L-TGA) is a congenital defect with not many probabilities of surgical correction in our country. Among the available therapeutic alternatives, the double switch surgery (DSS) offers a physiological

Solicitud de sobretiros: Víctor Raúl Castillo, MD, Fundación Cardiovascular de Colombia

Calle 155 A No 23-58, Floridablanca, Santander, Colombia.

Teléfono: (57-7) 6399292 Ext. 201 Fax: (57-7) 6392595 Correo electrónico: inv_pediatria@fcv.org

Recibido el 18/Enero/2005. Aceptado para publicación el 29/Abril/2005.

Este artículo está disponible en http://www.uady.mx/sitios/biomedic/revbiomed/pdf/rb051624.pdf

Vol.16/No.2/Abril-Junio, 2005 


\section{A Durán, VR Castillo, A Montero, C Villa-Roel.}

and safe correction for the infant in the short and long term.

Clinical presentation. Two cases of L-TGA with severe tricuspid regurgitation, one of them associated to Ebstein anomaly, are presented. Pulmonary banding was performed in both cases in preparation for the DSS.

Comments. The DSS is considered a novel and safe alternative because it offers an anatomic/ functional L-TGA correction and avoids the problems associated with the classic intervention. Although these two cases are the first succesfully performed in the Fundación Cardiovascular de Colombia (Bucaramanga - Colombia), they requiere a long follow-up period with special emphasis in the observation of the right atrial obstructive gradients and the tricuspid regurgitation behavior, both of which can produce venous congestive signs.

(Rev Biomed 2005; 16:95-98)

Key words: Levo-transposition of the great arteries, double switch surgery.

\section{INTRODUCCIÓN.}

La levo-transposición de grandes arterias (LTGA) es una cardiopatía congénita con pocas probabilidades de opción quirúrgica en nuestro medio. Sin embargo, a nivel mundial en los últimos 50 años se han desarrollado diferentes alternativas terapéuticas para su corrección, siendo la cirugía de doble switch (CDS) la mejor opción quirúrgica disponible, ya que ofrece una corrección fisiológica y segura para el infante en el corto y mediano plazo (1).

En el presente artículo se presentan dos casos de L-TGA con insuficiencia tricuspídea severa, uno de ellos asociado a anomalía de Ebstein. En ambos pacientes se realiza cerclaje de la arteria pulmonar como preparación a la CDS.

\section{PRESENTACIÓN DE LOS CASOS.}

\section{Caso 1.}

Preescolar masculino de 5 años con diagnóstico de L-TGA y anomalía de Ebstein desde el primer mes de vida (atrialización del 44\%). A los 4 años de edad desarrolla disnea con el ejercicio y se encuentra bloqueo atrioventricular completo e insuficiencia tricuspídea severa. En ventriculografía nuclear se encuentra fracción de eyección ventricular izquierda (FEVI) de 58\%. Se lleva a cirugía para colocación de marcapaso bicameral epicárdico y creación de cerclaje pulmonar. El gradiente final del cerclaje pulmonar es $54 \mathrm{mmHg}$ y la insuficiencia tricuspídea moderada con un gradiente de regurgitación de 34\%. Al año siguiente se programa para corrección total del L-TGA con circulación extracorpórea (CEC) y cardioplejia cristaloide de alta concentración de potasio a $28^{\circ} \mathrm{C}$. Utilizando la técnica de Senning para el switch auricular se corrige la anomalía de Ebstein con anillo de dacrón, plicando el segmento atrializado del ventrículo. Ante la presencia de insuficiencia mitral moderada, se realiza anuloplastía sobre el anillo valvular a nivel de cada comisura. Posteriormente se continúa con el switch de grandes vasos, según la técnica de Jattene, sin la realización de la maniobra de Lecompte. El tiempo de CEC es 271 minutos y el de isquemia 206 minutos. Finalmente se procede a calentar al paciente, corroborando adecuada captura del marcapaso. Se cierra por planos sin complicaciones y se traslada a la unidad de cuidado intensivo pediátrico (UCIP) donde se extuba en las primeras 24 horas. Como complicación postoperatoria presenta un síndrome de vena cava superior relacionado con un gradiente de $12 \mathrm{mmHg}$ antes de la entrada de las cavas a la neoaurícula, gradiente que posteriormente desciente a $9 \mathrm{mmHg}$ (desapareciendo el síndrome). Ante evidencia ecocardiográfica de adecuada contractilidad cardiaca, insuficiencia mitral leve a moderada y doble lesión tricuspídea leve con un gradiente máximo de 9 $\mathrm{mmHg}$, se da de alta con tratamiento oral anticongestivo y vasodilatador (furosemida y captopril).

En control a los dos meses posteriores a la cirugía no se encuentra gradiente obstructivo a la entrada de la neoaurícula, las válvulas atrioventriculares funcionan normalmente, se evidencia insuficiencia I-II

\section{Revista Biomédica}




\section{Corrección quirúrgica de L-transposición de grandes arterias.}

de la válvula mitral y grado I de la válvula tricuspídea, con un gradiente máximo de $5 \mathrm{mmHg}$; el septum interventricular se encuentra aún disquinético y la válvula aórtica con leve déficit para su cierre.

\section{Caso 2.}

Preescolar femenino de 5 años con antecedente de detección de soplo cardiaco grado IV/VI con adecuado crecimiento pondo-estatural desde los 31 meses de edad. Ecocardiograma revela L-TGA con leve displasia tricuspídea e índice de regurgitación de $21 \%$, insuficiencia mitral trivial, dilatación del ventrículo derecho y aurícula izquierda. Se inicia tratamiento con Captopril y un año después presenta insuficiencia tricuspídea severa, mitral leve y clase funcional III. Se realiza cerclaje de la arteria pulmonar, con lo que se resuelven los signos de falla cardiaca y mejora la arquitectura del ventrículo izquierdo. A los 5 años se realiza CDS: bajo CEC, hipotermia $\left(26^{\circ} \mathrm{C}\right)$ y cardioplejia cristaloide se realiza switch atrial con técnica de Senning, anuloplastia tricuspídea y switch de grandes vasos con la técnica de Jattene y la maniobra de Lecompte. El tiempo de CEC fue 272 minutos y el de isquemia 217 minutos. Sale a UCIP con soporte inotrópico de Epinefrina y Milrinone, siendo extubado durante las primeras 24 horas. Tuvo una hospitalización prolongada (16 días), debido a ritmo cardiaco nodal asociado a signos de falla cardiaca, manejado con infusión de Dobutamina y marcapaso transitorio. Control ecocardiográfico postoperatorio revela contractilidad cardiaca normal, con insuficiencia tricuspídea moderada y gradiente obstructivo del túnel de la aurícula derecha de 9 mmHg. Egresa a los 15 días de hospitalizado con tratamiento con Digoxina, Captopril y Furosemida, encontrándose en clase funcional I. A los tres meses post-cirugía, persiste asintomático, se encuentra gradiente obstructivo supravalvular pulmonar hasta de $60 \mathrm{mmHg}$ y se programa para dilatación con balón y probable colocación de stent.

\section{DISCUSIÓN.}

Los dos casos presentados en el presente artículo, son los primeros realizados exitosamente en la Fundación Cardiovascular de Colombia (Bucaramanga-Colombia). Sin embargo, requieren de seguimiento en el largo plazo con especial énfasis en el comportamiento de los gradientes obstructivos de la aurícula derecha y de la insuficiencia tricuspídea que pueden llevar a signos de congestión venosa.

La CDS se considera una alternativa segura y novedosa para los pacientes con transposición congénita de grandes arterias desde 1989, con buenos resultados a mediano plazo (10 años) (1). Esta cirugía además de ofrecer una corrección anatómica y funcional de la L-TGA, evita los problemas asociados a la corrección clásica: falla ventricular sistémica hasta en el $40 \%$ de los pacientes dentro de los tres años posteriores a una corrección clásica para L-TGA y muerte hasta en un 52\% de los pacientes en el largo plazo $(2,3)$. Estas cifras explican el que varios autores consideren la corrección clásica como no satisfactoria, no sólo por su alta mortalidad, sino por la necesidad de reintervención quirúrgica (cambio de válvula tricuspídea) en el $80 \%$ de los pacientes (4).

Desde la primera descripción de la CDS realizada por el Doctor Roger Mee en 1989, se han reportado casos con resultados alentadores en la literatura médica universal. Ibawi y colaboradores fueron los primeros en reportar dos casos exitosos en 1990. Posteriormente se han reportado muy buenos resultados en el corto y mediano plazo con este tipo de cirugía, con mortalidades que oscilan entre $0-9 \%$ y mínimas complicaciones postoperatorias (5). Sin embargo, se necesitan estudios prospectivos para evaluar el verdadero impacto de esta cirugía sobre la calidad de vida de pacientes con L-TGA.

\section{REFERENCIAS.}

1.- lbawi MN, Ocampo CB, Allen BS, Barth MJ, Roberson DA, Chiemnongkoltip P, et al. Intermediate results of the anatomic repair for congenitally corrected transposition. Ann Thorac Surg 2002; 73:594-600.

2.- Sano T, Riesenfeld T, Kart TR, Wilkinson JL. Intermediate term outcome after intracardiac repair of 


\section{A Durán, VR Castillo, A Montero, C Villa-Roel.}

associated cardiac defects in patients with atrioventricular and ventriculoarterial discordance. Circulation. 1995; 92:272-8.

3.- Yeh T, Connelly MS, Coles JG, Webb GD, McLaughlin PR, Freedom RM, et al. Atrioventricular discordance: Results of repair in 127 patients. J Thorac Cardiovas Surg 1999; 117:1190-211.

4.- Termignon JL, Leca F, Vouhé PR, Vernant F, Bical OM, Lecompte Y, et al. «Classic » repair of congenitally corrected transposition and ventricular septal defect. Ann Thorac Surg. 1996; 62:199-206.

5.- Imamura M, Drummond- Webb JJD, Murphy DJ, Prieto LR, Latson LA, Flamm SD, et al. Results of the Double Switch Operation in the Current Era. Ann Thorac Surg 2000; 70:1005. 